\title{
Psicooncología
}

ISSN: 1696-7240

\section{Entrevista semi-estructurada para la evaluación de la conciencia de en- fermedad oncológica E-CEO. Estudio de fiabilidad sobre una muestra de pacientes con enfermedad avanzada}

\author{
María Gorety Pazos González¹; María José Carreira Vida2²*; Jorge García Fernández ${ }^{3}$
}

Recibido: 2 de julio de 2018 / Aceptado: 14 de diciembre de 2018

Resumen: Objetivo: La comunicación entre pacientes oncológicos y profesionales de la salud se considera uno de los aspectos más importantes del cuidado, especialmente en fases avanzadas de la enfermedad. La conciencia de enfermedad consistiría en un proceso dinámico y cambiante en el tiempo, influenciado por la cantidad y calidad de la información y el significado que cada sujeto le asigna. En el presente trabajo se describe el proceso de diseño, validación y cálculo de la fiabilidad de una entrevista semiestructurada (Entrevista sobre la Conciencia de Enfermedad Oncológica E-CEO). Este instrumento, escasamente invasivo y no revelador de información, permite situar a los sujetos a lo largo de un continuum en base a su percepción de severidad de la enfermedad. Método: En una primera fase se realizó una entrevista abierta sobre 15 pacientes y se elaboró un listado de expresiones clave, que fueron agrupadas en cinco categorías. En una segunda fase se aplicó un modelo de entrevista semiestructurada a 42 pacientes ingresados con diagnóstico de enfermedad oncológica avanzada (estadio clínico IV) en la Unidad de Hospitalización de Oncología del Hospital de A Coruña. Resultados: La fiabilidad obtenida entre diferentes observadores muestra que se trata de un instrumento de elevada calidad (Kappa=0,70). Los datos reflejan una discrepancia amplia entre el estado de salud de los pacientes y su propia consideración. Más del 50\% consideraron su enfermedad "controlada". Conclusiones: Se trata de un instrumento de sencilla aplicación que permitirá en futuras investigaciones profundizar en el estudio de los procesos de integración de información en este tipo de pacientes.

Palabras clave: cáncer, conciencia de enfermedad, entrevista semiestructurada.

[en] Semi-structured interview for the evaluation of oncological disease awareness E-CEO. Reliability study on a sample of patients with advanced disease

\footnotetext{
Abstrac: Objective: Communication between cancer patients and health professionals is considered one of the most important aspects of care, especially in advanced stages of the disease. Disease awareness, consists of a dynamic and evolving process over time, influenced by the quantity and quality

1 María Gorety Pazos González. Médico Servicio Oncología Médica. Xerencia de Xestión Integrada A Coruña. E-mail: maria.gorety.pazos.gonzalez@sergas.es

2 María José Carreira Vidal Psicóloga Clínica. Diplomada en Enfermería. Xerencia de Xestión Integrada A Coruña. E-mail: mcarvid@icloud.com

3 Jorge García Fernánde. Facultativo Especialista de Área de Psicología Clínica. Xerencia de Xestión Integrada A Coruña.

E-mail: jorge.garcia.fernandez@sergas.es

* Dirección de correspondencia: María José Carreira Vidal. Hospital Universitario de A Coruña. Departamento: Oncología 9 $9^{\mathrm{a}}$ planta, As Xubias, 84 - 15006 A Coruña. E-mail: mcarvid@icloud.com
} 
of information and significance that each individual assigns. In this paper, the process for the design, validation and reliable calculation of a semi-structured interview (Interview on the Consciousness of Oncological Disease E-CEO) is described. This process is sparingly intrusive, non-disclosing of information and makes it possible to place an individual along a continuum according to their degree of perception of disease severity. Method: In an initial phase, an open interview was conducted with 15 patients and a list was prepared of key expressions, which were then grouped into five categories. In a second phase, the semi-structured interview model was applied to 42 patients admitted with a diagnosis of advanced oncological disease (clinical stage IV) in the Oncology Hospitalization Unit of the Hospital of A Coruña. Results: The reliability obtained among observers shows that it is a highquality instrument $(\mathrm{Kappa}=.70)$. The data reflects a wide discrepancy between the health status of the patients and their own perception. More than 50\% consider their illness "controlled". Conclusions: This is a simple application that will allow future in-dept research into the study of information integration processes for these types of patients.

Keywords: cancer, disease awareness, semi-structured interview.

Sumario. 1. Introducción 2. Método 3. Resultados 4. Discusión y conclusiones 5. Referencias bibliográficas.

Cómo citar: Pazos González MG, Carreira Vidal MJ, García Fernández J. Entrevista semiestructurada para la evaluación de la conciencia de enfermedad oncológica E-CEO. Estudio de fiabilidad sobre una muestra de pacientes con enfermedad avanzada. Psicooncología 2019;16:11-23. doi: 10.5209/PSIC.63645.

\section{Introducción}

La comunicación entre pacientes con cáncer y profesionales de la salud se ha identificado como uno de los aspectos más importantes del cuidado, especialmente en fases avanzadas de la enfermedad ${ }^{(1)}$. Una comunicación centrada en el paciente, que reconozca las necesidades de información a lo largo de la trayectoria de su enfermedad y adaptada en cantidad, contenido, forma y ritmo contribuye al bienestar psicosocial del enfermo. Se encuentra además asociada con una mayor satisfacción y adherencia a los tratamientos, resultando por ello fundamental para ofrecer cuidados de alta calidad ${ }^{(2-5)}$.

En las últimas décadas se han producido cambios notables en las actitudes de los sanitarios y de los pacientes sobre la accesibilidad a la información de su diagnóstico ${ }^{(6-11)}$. No obstante, parece existir una gran variabilidad en lo que respecta a la información declarada por los pacientes de diferentes países ${ }^{(12)}$ y en diferentes puntos de España ${ }^{(13,14)}$. La posibilidad de que éstos puedan ejercer un papel activo a lo largo del proceso de su enfermedad requiere tener en consideración además del diagnóstico, el estado de la evolución de la enfermedad en un momento determinado.

El proceso de integrar datos, en ocasiones contradictorios, proveniente de distintas fuentes (señales físicas, información médica, lecturas, amigos y familiares...) es un fenómeno complejo. Diferentes estudios ponen de manifiesto la presencia de mecanismos que actúan como filtro entre la información externa y las declaraciones que posteriormente realizan los pacientes sobre dichos contenidos ${ }^{(8,10,11,15-19)}$. La integración a nivel emocional de la información consiste en un proceso no lineal ${ }^{(20)}$ donde las necesidades, expectativas, factores culturales y habilidades de afrontamiento influyen decisivamente ${ }^{(21)}$. 
El término conciencia de enfermedad se ha empleado comúnmente para definir de forma genérica el grado de conocimiento que un paciente declara acerca de su estado de salud. Morasso et al. (21) la definen como un proceso dinámico y cambiante en el tiempo, influenciado por la cantidad y calidad de la información ofrecida al paciente y el significado que éste le asigna. Existen numerosas aproximaciones hacia la evaluación de este constructo mediante cuestionarios y escalas ${ }^{(22)}$. En el ámbito de la oncología, al igual que en otros campos como el daño cerebral ${ }^{(23)} \mathrm{O}$ la esquizofrenia ${ }^{(24)}$, la posibilidad de emplear entrevistas semiestructuradas se ha considerado una herramienta de elección que posibilita un acercamiento escasamente invasivo y no revelador de información $n^{(9,19,21,25-30)}$.

La obtención de una medida de la conciencia de enfermedad exige obtener la opinión del paciente sobre su estado de salud y contrastarla con su situación clínica valorada por un profesional. En 1997, Morasso et al. ${ }^{(21)}$ elaboraron un instrumento multidimensional con aspectos informativos y emocionales que permitirían situar a los sujetos en una escala de siete niveles de conciencia de enfermedad en base a la congruencia observada por el evaluador entre las diferentes dimensiones. Los estudios de fiabilidad aconsejaron reducir esta clasificación a tres niveles: pacientes conscientes, pacientes con mecanismos de defensa y parcialmente no conscientes de la enfermedad. Estudios posteriores concluyeron con similares dificultades de validez a la hora de comparar esta escala con las impresiones clínicas de los profesionales ${ }^{(31)}$. Se trata de un instrumento amplio y de aplicación compleja que requiere que el evaluador conozca previamente la situación clínica del paciente. A pesar de que se consideran aspectos informativos sobre diagnóstico, pronóstico y tratamiento, estas variables no se especifican en la valoración final realizada con este instrumento.

La posibilidad de realizar la evaluación de estos aspectos mediante las impresiones de familiares o profesionales en contacto habitual con los pacientes se ha tenido en cuenta en algunos trabajos ${ }^{(32,33)}$. Corli et al. aplicaron una entrevista estructurada a cuidadores y pacientes acerca de aspectos informativos y actitudinales encontrando un elevado nivel de concordancia entre las manifestaciones de ambos. Dicho instrumento, al igual que el anterior, ofrece datos acerca de la conciencia de padecer cáncer, otra enfermedad, o incluso ninguna en concreto, sin especificar niveles en cuanto a la progresión de la enfermedad ni datos acerca de la fiabilidad de dichas medidas. Por el contrario, en el estudio de Burns et al. ${ }^{(33)}$ se obtuvieron amplias discrepancias entre la consideración de la enfermedad de diferentes díadas (pacientecuidador) evaluadas.

Andruccioli et al. ${ }^{(34)}$ realizaron un estudio aplicando una entrevista breve sobre el diagnóstico o pronóstico, obteniendo correlaciones moderadas entre las declaraciones realizadas por los pacientes y la opinión de los psicólogos, enfermeras, familiares y médicos sobre el grado de conciencia en relación con su situación médica. Nuevamente la clasificación ofrecida por la escala es dicotómica (consciente / no consciente) y tampoco se ofrecen normas específicas para el análisis del discurso de los pacientes. Esta última cuestión es tenida en cuenta por otros trabajos $^{(19,35)}$ aunque únicamente ofrecen algunas orientaciones sobre los criterios de valoración empleados en el análisis de las respuestas sin que se realizasen estudios de fiabilidad de las clasificaciones empleadas. Estas importantes limitaciones que se extienden al resto de trabajos que emplean este tipo de entrevista ${ }^{(36,37)}$, comprometen su calidad metodológica y dificultan la realización de comparaciones entre los distintos estudios. 
En definitiva, diferentes instrumentos que han abordado esta cuestión resultan en ocasiones complejos y limitados en cuanto a los datos que ofrecen, así como de fiabilidad cuestionable dada la ausencia de normas específicas de interpretación de las respuestas de los sujetos.

En nuestra opinión, la manera conveniente de medir esta variable precisa del diseño de una herramienta que permita esencialmente una valoración cuidadosa del tipo y grado de enfermedad considerado por los enfermos. Decidir si ésta es o no congruente, así como su distancia con el estado clínico "real" resultaría una tarea más sencilla mediante su comparación con los datos objetivos acerca de la progresión de la patología oncológica.

Con este objetivo nos hemos planteado, en primer lugar, la elaboración de una entrevista semiestructurada centrada en aspectos de diagnóstico y pronóstico, donde ambos formen parte de una misma dimensión, desde la ausencia de enfermedad hasta la fase de enfermedad avanzada terminal. Un instrumento capaz de transformar lo que el paciente relata acerca de su enfermedad, independientemente de la coherencia con su estado físico objetivo, en distintas categorías de severidad de su patología. En segundo lugar, para analizar las propiedades de este instrumento, hemos realizado un análisis de la fiabilidad sobre una muestra de pacientes con enfermedad avanzada.

\section{Método}

Tras la aprobación de la realización de este estudio por parte del Comité Ético de Investigación del centro hospitalario y con el correspondiente consentimiento informado de los participantes, se seleccionaron al azar 62 pacientes ingresados en la Unidad de Hospitalización de Oncología Médica del Hospital de A Coruña. Todos los sujetos tenían diagnóstico de enfermedad oncológica avanzada (estadio clínico IV). Quedaron excluidos aquellos que presentaron diagnóstico de trastorno psiquiátrico grave o trastorno mental orgánico.

En una primera fase del estudio se seleccionaron 15 pacientes que fueron entrevistados con la única finalidad de comprobar la idoneidad de las preguntas y recoger una muestra discursiva con la que elaborar la clasificación de categorías posteriormente empleada. En la segunda fase dirigida al análisis de la fiabilidad, se invitó a participar a 47 individuos de los cuales accedieron a colaborar 45 siendo 3 eliminados en la fase de entrevista por alteraciones de su estado cognitivo. Finalmente 42 sujetos participaron en esta fase, con una media de edad de 57 años, con diversas patologías, tiempo de evolución y tipos de tratamiento previos. Presentamos en la tabla 1 información acerca de diferentes datos clínicos y variables sociodemográficas.

En una primera fase se llevó a cabo una entrevista individual a 15 pacientes en la que se les pedía que hablaran abiertamente de su enfermedad desde el comienzo de ésta hasta el momento actual. Se transcribieron literalmente las narraciones de cada paciente. Un grupo de cinco expertos (un psicólogo, dos enfermeras y dos oncólogos), identificaron diferentes términos frecuentemente utilizadas por los pacientes para referirse a su enfermedad, la gravedad percibida y al grado de evolución de la misma y se elaboró un listado de expresiones clave. Las respuestas obtenidas se agruparon, siguiendo un procedimiento de consenso ${ }^{(39)}$, en cinco categorías en función del nivel 
Tabla 1. Características demográficas y clínicas de la muestra $(n=42)$

\begin{tabular}{|c|c|}
\hline \multirow[b]{2}{*}{ Media \pm DT } & \multirow[b]{2}{*}{$57 \pm 10$ (Rango $25-76)$} \\
\hline & \\
\hline \multicolumn{2}{|l|}{ Sexo } \\
\hline Hombre & $26(61,9 \%)$ \\
\hline Mujer & $16(38,1 \%)$ \\
\hline \multicolumn{2}{|l|}{ Nivel educativo: } \\
\hline Educación primaria & $18(42,9 \%)$ \\
\hline Educación secundaria & $11(26,2 \%)$ \\
\hline Formación Profesional & $5(12 \%)$ \\
\hline Universidad & $8(19 \%)$ \\
\hline \multicolumn{2}{|l|}{ Tipo de Tumor: } \\
\hline Pulmón & $20(47,6 \%)$ \\
\hline Mama & $6(14,3 \%)$ \\
\hline Colorrectal/Digestivo & $9(21,4 \%)$ \\
\hline Urológico & $2(4,8 \%)$ \\
\hline Otro & $5(12 \%)$ \\
\hline \multicolumn{2}{|l|}{ Tipo de tratamiento: } \\
\hline Quimioterapia & $31(73,8 \%)$ \\
\hline Radioterapia & $2(4,8 \%)$ \\
\hline Control de síntomas & $7(16,7 \%)$ \\
\hline Hormonoterapia & $0(0 \%)$ \\
\hline Combinado & $1(2,4 \%)$ \\
\hline \multicolumn{2}{|l|}{ Performance Estatus (Escala funcional ECOG/OMS) (38) } \\
\hline Media \pm DT & $2,5 \pm 0,86$ (rango $1-4)$ \\
\hline \multicolumn{2}{|l|}{ Tiempo del diagnóstico (meses) } \\
\hline Media \pm DT & $20,9 \pm 35,95$ \\
\hline Rango & $1-192$ \\
\hline
\end{tabular}

DT=Desviación típica. 
de progresión de la enfermedad, de menor a mayor grado: ausencia de enfermedad oncológica, enfermedad oncológica diagnosticada, enfermedad a tratamiento controlada, enfermedad en progresión y enfermedad avanzada sin tratamiento antitumoral. Se desarrolló un primer borrador para una entrevista semiestructurada formado por preguntas que podían ser adaptadas o reformuladas por el investigador en función del contexto. Dicho borrador se puso a prueba en cinco pacientes y se modificó para hacer la entrevista más fluida y comprensible. Finalmente, se diseñó una versión final compuesta por 5 preguntas abiertas. En el ANEXO I se incluyen las instrucciones y en el ANEXO II el protocolo de valoración.

En una segunda fase se aplicó la entrevista a 42 pacientes. Cada entrevista, realizada por una psicóloga componente del equipo investigador, fue registrada en audio y puntuada de forma independiente por 4 evaluadores seleccionados por su escasa experiencia en el ámbito de la oncología (una enfermera de salud mental, una enfermera matrona, un residente de psicología clínica y una estudiante de posgrado). Para ello se les proporcionó exclusivamente las instrucciones que figuran en el ANEXO II incluyendo algunos ejemplos para la valoración en cada categoría.

\section{Resultados}

Los cuatro evaluadores situaron a cada uno de los 42 sujetos en una de las cinco categorías descritas sumando un total de 168 clasificaciones. De ellos, ninguno fue clasificado en la categoría A, 12 evaluaciones (7,14\%) se situaron en la B, $84(50 \%)$ en la C, 67 (39,8 \%) en la D y $5(2,9 \%)$ en la E (Tabla2). Tal como hemos expuesto anteriormente la totalidad de los sujetos presentaba una enfermedad en progresión sin opciones de tratamiento oncológico y en situación de ingreso con fines paliativos. De hecho, ninguno de los pacientes sobrevivió más de 20 semanas tras la realización del estudio.

Tabla 2. Resultados de la aplicación de las categorías por los 4 observadores

\begin{tabular}{|l|r|r|}
\hline Categoría & $\mathrm{N}$ & Porcentaje \\
\hline A-No conoce diagnóstico & 0 & 0 \\
\hline B-Conoce diagnóstico de tumor & 12 & $7,14 \%$ \\
\hline C-Diagnóstico tumor maligno & 84 & $50 \%$ \\
\hline D-Tumor maligno avanzado & 67 & $39,8 \%$ \\
\hline E- Enfermedad terminal & 5 & $2,9 \%$ \\
\hline
\end{tabular}

Se empleó el programa informático de acceso libre para análisis epidemiológico y estadístico de datos Epidat 4.2 para realizar el análisis de la concordancia mediante el coeficiente Kappa sobre varios observadores y más de dos categorías. La categoría A no fue tenida en cuenta en el análisis al no disponer de ninguna clasificación 
sobre ella. El índice kappa global obtenido fue de 0,70 considerado como un nivel de fiabilidad elevado (Tabla 3). Las categorías B y D presentaron una concordancia considerada igualmente como sustancial, la categoría $\mathrm{C}$ una concordancia moderada y finalmente la categoría $\mathrm{E}$ con un valor que deberíamos considerar como bajo o discreto $^{(39,40)}$.

Tabla 3: Concordancia por categorías y total entre los evaluadores

\begin{tabular}{|l|c|c|c|c|c|}
\hline Categoría & Kappa & $\mathrm{IC}^{*}(95,0 \%)$ & Estadístico Z & Valor $\mathrm{p}$ & \\
\hline B & 0,8205 & 0,5078 & 1,1315 & 13,0252 & 0,0000 \\
\hline C & 0,6984 & 0,5455 & 0,8510 & 11,0870 & 0,0000 \\
\hline D & 0,7104 & 0,5599 & 0,8606 & 11,2766 & 0,0000 \\
\hline E & 0,3816 & $-0,4619$ & 1,2155 & 6,0576 & 0,0000 \\
\hline Kappa global & 0,7015 & 0,5686 & 0,8341 & 14,2271 & 0,0000 \\
\hline
\end{tabular}

* Intervalo de confianza.

\section{Discusión y conclusiones}

A pesar de la aparente simplicidad del estudio, alcanzar un índice de concordancia considerado como aceptable ha resultado un proceso laborioso. Se ha seleccionado un método exigente de análisis de la concordancia considerando, por un lado, la escala como estrictamente nominal (sin ajustes ponderados en las categorías adyacentes), $\mathrm{y}$, por otro lado, empleando más de dos observadores, que si bien garantiza en mayor medida la calidad del estudio, ha dificultado sin duda la obtención de un mayor índice de fiabilidad. Los datos obtenidos apoyan la posibilidad de utilizar esta entrevista con un nivel bajo de entrenamiento, e incluso, tal como hemos realizado en este estudio, la posibilidad de ser empleada por evaluadores sin experiencia en el ámbito oncológico.

Este trabajo cuestiona los estudios previos que dan por hecho la capacidad de los clínicos para valorar el nivel de conocimiento del paciente sobre su enfermedad, sin emplear un procedimiento específico, ni poner en cuestión su fiabilidad ${ }^{(19,33,35-37)}$. En nuestro caso, tras haber realizado un importante esfuerzo en este sentido, todavía los resultados son mejorables. Especialmente las instrucciones elaboradas y consensuadas para codificar la "enfermedad terminal" no han alcanzado un grado de acuerdo elevado.

Es muy probable también que existan importantes diferencias incluso en nuestro país a la hora de hacer referencia a los términos empleados en la codificación, por lo cual entendemos que este modelo de entrevista expuesto debería ser ajustado tanto a las características idiomáticas como culturales de las diferentes zonas geográficas, así como a la evolución del propio lenguaje con el paso del tiempo. El empleo de una muestra homogénea en cuanto a la situación de enfermedad (pacientes en 
estadío IV hospitalizados bajo tratamiento paliativo) ha restado información sobre el comportamiento del instrumento diseñado en otros niveles de severidad. En nuestra opinión sería conveniente mejorar las cuestiones mencionadas en futuras investigaciones.

Deseamos también realizar algunos comentarios en vista de los datos disponibles que informan, como resulta habitual en la literatura, de la presencia de amplias discrepancias entre la valoración médica y la declaración de los pacientes acerca de su estado de salud(12-14,32,41,42). En el presente estudio, el 97\% de las clasificaciones realizadas por los observadores situaron a los sujetos en niveles inferiores de conciencia de severidad con respecto a su situación clínica. Incluso en ocasiones esta distancia resultó considerable (dos niveles), con declaraciones del 50\% de los pacientes en estado terminal que informaron sobre el padecimiento de una enfermedad controlada. Esto supone una discrepancia mayor de la habitual en otros trabajos, que atribuimos, al menos en parte, a la mayor precisión requerida en el proceso de codificación de las verbalizaciones de los pacientes. Por otro lado, la mayoría de las investigaciones realizan valoraciones dicotómicas ("consciente" o "no consciente") o en tres niveles añadiendo la categoría de "parcialmente consciente", lo cual incrementa las posibilidades de coincidencia con respecto a una escala de cinco categorías.

Frecuentemente se apunta como causa de estas discrepancias a la ausencia de oportunidades para resolver necesidades informativas no satisfechas, sin embargo, como ya comentamos, es conocido que no existe comúnmente una relación estrecha entre la información ofrecida y la manifestada por los pacientes ${ }^{(43)}$. Resulta llamativo en la muestra estudiada, que los sujetos no sean conscientes de la gravedad de su condición médica aún a pesar de disponer de amplias oportunidades para tratar cuestiones informativas. De hecho, la percepción de los profesionales es la de haber ofrecido mayor información que la que los pacientes declaran.

Sería deseable en el futuro avanzar, con la ayuda de este tipo de estrategias de evaluación, en el conocimiento de estos mecanismos moduladores de las señales propias y ajenas que nos informan sobre la severidad de nuestras circunstancias médicas. Esperamos contribuir con ello a la mejora de la calidad de la investigación sobre la búsqueda de explicaciones acerca de estos importantes aspectos que afectan a la calidad de la asistencia y al bienestar emocional de los pacientes.

\section{Referencias bibliográficas}

1. Steinhauser KE, Christakis NA, Clipp EC, McNeilly M, Grambow S, Parker J, et al. Preparing for the end of life: preferences of patients, families, physicians, and other care providers. J Pain Symptom Manage 2001;22:727-37

2. Epstein RM, Street RL. [Internet] Patient-Centered Communication in Cancer Care: Promoting Healing and Reducing Suffering. Bethesda (MD): National Cancer Institute. 2007.

3. Innes S, Payne S. Advanced cancer patients' prognostic information preferences: a review. Palliat Med 2009; 23: 29-39.

4. Zachariae R, Pedersen CG, Jensen AB, Ehrnooth E, Rossen PB, Maase H. Association of perceived physician communication style with patient satisfaction, distress, cancer-related self-efficacy, and perceived control over the disease. Br J Cancer 2003; 88: 658-65. 
5. Stewart M, Brown JB, Boon H Galajda J, Meredith L, Sangster M. Evidence on patientdoctor communication. Cancer Prev Control 1999; 3:25-30.

6. Degner LF, Kristjanson LJ, Bowman D, Sloan JA, Carriere KC, O' Neil J, et al. Information needs and decisional preferences in women with breast cancer. JAMA. 1997; 277:1485-92.

7. Friis LS, Elverdam B, Schmidt KG. The patient's perspective. A qualitative study of acute myeloid leukaemia patients' need for information and their information-seeking behaviour. Support Care Cancer. 2003;11:162-70.

8. Kaplowitz SA, Campo S, Chiu WT. Cancer patients' desires for communication of prognosis information. Health Commun 2002; 14:221-41

9. Leydon GM, Boulton M, Moynihan C, Jones A, Mossman J, Boudioni M, et al. Cancer patients'information needs and information seeking behaviour: in depth interview study. BMJ 2000; 320:909-13.

10. Muthu Kumar D, Symonds RP, Sundar S, Ibrahim K, Savelyich BS, Miller E. Information needs of Asian and white British cancer patients and their families in Leicestershire: A cross-sectional survey. Br J Cancer 2004; 90:1474-8.

11. Pimentel FL, Ferreira JS, Vila Real M, Mesquita NF, Maia-Goncalves JP. Quantity and quality of information desired by Portuguese cancer patients. Support Care Cancer 1999; 7:407-12

12. Nuñez Olarte JM. Información al paciente en situación terminal: diferencias entre las publicaciones anglosajonas y la experiencia diaria en España. Med Paliat 2013; 21: 11320.

13. Centeno-Cortes C, Nuñez-Olarte JM. Estudios sobre la comunicación del diagnóstico del cáncer en España. Med Clin 1998; 110:744-50.

14. Estapé J, Palombo H, Hernandez E, Daniels M, EStapé T, Grau JJ, et al. Cancer diagnosis disclosure in a Spanish hospital. Ann Oncol 1992; 3: 451-4.

15. Fallowfield L, Jenkins V. Communicating sad, bad, and difficult news in medicine. Lancet 2004;363:312-9

16. Gordon EJ, Daugherty CK. 'Hitting you over the head': oncologists' disclosure of prognosis to advanced cancer patients. Bioethics 2003;17:142-68.

17. Seo M, Tamura K, Shijo H, Morioka E, Ikegame C, Hirasako K. Telling the diagnosis to cancer patients in Japan: attitude and perception of patients, physicians and nurses. Palliat Med 2000; 14:105-10.

18. Watson M, Greer S, Blake S, Shrapnell K. Reaction to a diagnosis of breast cancer: relationship between denial, delay, and rates of psychological morbidity. Cancer 1984; 53: 2008-12.

19. Caruso A, Di Francesco B, Pugliese P, Cinanni V, Corlito A. Information and awareness of diagnosis and progression of cancer in adult and elderly cancer patients. Tumori 2000;86:199-203.

20. Field D, Copp G. Communication and awareness about dying in the 1990s. Palliat Med 1999; 13: 459-68.

21. Morasso G, Alberisio A, Capelli M, Rossi C, Baracco G, Costantini M. Illness awareness in cancer patients: a conceptual framework and a preliminary classification hypothesis. Psychooncology 1997; 6: 212-7.

22. Applebaum AJ, Kolva EA, Kulikowski JR, Jacobs JD, DeRosa A, Lichtenthal WG, Olden ME, Rosenfeld B, Breitbart W. Conceptualizing prognostic awareness in advanced cancer: a systematic review. J Health Psychol 2014;19:1103-19. 
23. Malouf T, Langdon R, Taylor A. The Insight Interview: a new tool for measuring deficits in awareness after traumatic brain injury. Brain Injury 2014; 28: 1523-41.

24. Camprubi N, Almela A, Garre-Olmo J. Propiedades psicométricas de la validación española de la Insight Scale. Actas Españolas de Psiquiatría 2008; 36: 323-30.

25. Iconomou G, Viha A, Koutra A, Vagenakis AG, Kalofonos HP. Information needs and awaraness of diagnosis in patients with cancer receiving chemotherapy: A report from Greece. Palliat Med 2002;16:315-21

26. Chominov HM, Tataryn DJ, Wilson KG, Enns M, Lander S. Prognosis awareness and the terminally ill. Psychosomatic. 2000; 41: 500-4.

27. Pronzato P, Bertelli G, Losardo P, Landucci M. What do advanced cancer patients know of their disease? A report from Italy. Support Care Cancer 1994; 2: 242-4.

28. Nord C, Mykletun A, Fossa SD. Cancer patients' awareness about their diagnosis: A population-based study. J Public Health Med 2003;25: 313-7.

29. Helft PR, Hlubocky F, Wen M, Daugherty CK. Associations among awareness of prognosis, hopefulness, and coping in patients with advanced cancer participating in phase I clinical trials. Support Care Cancer. 2003;11:644-51.

30. Centeno-Cortes C, Nuñez-Olarte JM. Questionning diagnosis disclosure in terminal cáncer patients: A prospective study evaluating patients responses. Palliat Med 1994;8:39-44.

31. Andruccioli J, Montesi A, Di Leo S, Sarti D, Turci P, Pittureri C, et al . Illness awareness in hospice: application of a semi-structured interview. Am J Hosp Palliat Care 2009;26:384-91.

32. Corli O, Apolone G, Pizzuto M, Cesaris L, Cozzolino A, Orsi L, et al. Illness awareness in terminal cancer patients: an Italian study. Palliat Med 2009;23:354-9.

33. Burns CM, Broom DH, Smith WT, Dear K, Craft PS. Fluctuating awareness of treatment goals among patients and their caregivers: A longitudinal study of a dynamic process. Support Care in Cancer. 2007;15:187-96

34. Andruccioli J, Montesi A, Raffaeli W, Monterubbianesi MC, Turci P, Pittureri C, et al. Illness awareness of patients in hospice: psychological evaluation and perception of family members and medical staff. J Palliat Med 2007;10:741-8.

35. Costantini A, Grassi L, Picardi A, Brunetti S, Caruso R, Nanni MG, et al. Awareness of cancer, satisfaction with care, emotional distress, and adjustment to illness: an Italian multicenter study. Psychooncology 2015;24:1088-96. doi: 10.1002/pon.3768

36. Gattellari M, Butow PN, Tattersall MH, et al. Misunderstanding in cancer patients: Why shoot the messenger? Ann Oncol 1999;10:39-46.

37. Johnston G, Abraham C. Managing awareness: Negotiating and coping with a terminal prognosis. Inter J Palliat Nurs 2000; 6: 485-94.

38. Karnofsky DA, Burchenal JH. The clinical evaluation of chemotherapeutic agents in cancer. En: CM Macleod (ed.). Evaluation of chemotherapeutic agents. New York: Columbia University Press, 1949; p. 191-205.

39. Lynn MR. Determination and quantification of content validity. Nurs Res 1986;35:382-5.

40. Landis J, Koch G. The measurement of observer agreement for categorical data. Biometrics 1977; 33:159-74.

41. Martos-Gutiérrez JA, Montoro-Lorite M, Mundet-Pons L. Quality of diagnosis information given to terminal cancer patients. Enferm Clin 2016; 26:344-50. doi: 10.1016/j.enfcli.2016.07.006

42. Craft PS, Burns CM, Smith WT, Broom DH. Knowledge of treatment intent among patients with advanced cancer: a longitudinal study. Eur J Cancer Care 2005;14:417-25.

43. Hagerty RG, Butow PN, Ellis PM, Dimitry S, Tattersall MH. Communicating prognosis in cancer care: a systematic review of the literature. Ann Oncol 2005;16:1005-53. 


\section{ANEXO I \\ INSTRUCCIONES PARA DIRIGIR LA ENTREVISTA}

"Me gustaría que habláramos sobre la enfermedad que le han diagnosticado en el ... (indicar órgano para centrar la conversación) y que le están tratando en... (consulta/ planta) antes de este ingreso. Si la conversación le cansa o le molesta paramos cuando quiera. Por favor, responda brevemente.

1) ¿Puede explicarme de que se trata o en qué consiste su enfermedad?

Pregunta dirigida a descubrir el grado de conocimiento sobre el diagnóstico de enfermedad oncológica.

2) ¿Cómo ha sido la evolución de la enfermedad desde que conoció el diagnostico hasta ahora?

Pregunta que evalúa el conocimiento de la severidad de la enfermedad y/o de la fase evolutiva. Esta pregunta contribuye a aclarar el grado de sospecha de malignidad en aquellos pacientes en los que la respuesta anterior no es positiva.

3) ¿Cuál es la situación de esta enfermedad de la que venimos hablando, en este momento?

Pretende averiguar si el paciente conoce su situación actual y si intuye un posible cambio de situación clínica hacia estadio terminal o proximidad de la muerte (en aquellos casos que así sea).

Nota: es interesante indicar el órgano de origen al hacer la pregunta por qué si no puede centrarse la conversación en la complicación más reciente o el síntoma actual. Ayuda a centrar la pregunta.

4) ¿Puede explicarme en qué consiste el tratamiento que recibe en este momento para esa enfermedad; hablamos del tratamiento propio/especifico?

La pregunta evalúa el grado de conocimiento de los tratamientos que está recibiendo o ha recibido.

5) ¿Cuál es el objetivo de ese tratamiento? ¿Qué resultados espera usted de este tratamiento del que me habla?

Evalúa si paciente conoce que el tratamiento tiene intención paliativa, aunque incluya quimioterapia. En caso de ser únicamente control sintomático, evalúa si lo sabe. 


\section{ANEXO II \\ CATEGORÍAS DE CONCIENCIA DE ENFERMEDAD EN PACIENTES ONCOLÓGICOS CON ENFERMEDAD AVANZADA}

Según la información aportada por el paciente en las preguntas de la entrevista podríamos encuadrarlo en una de las siguientes 5 categorías

Categoría A: El paciente no refiere conocer el diagnóstico de enfermedad neoplásica. Las respuestas obtenidas no permiten aclarar el grado de conocimiento del diagnóstico y el pronóstico, o bien orientan directamente a un desconocimiento de la enfermedad actual

Categoría B: El paciente conoce el diagnóstico de enfermedad tumoral. Las respuestas obtenidas permiten afirmar que conoce la existencia de un tumor, pero no son suficientes para afirmar que conoce el diagnóstico de malignidad o bien orientan abiertamente a que lo desconoce.

Categoría C: El paciente conoce el diagnóstico de enfermedad tumoral maligna. En las respuestas el paciente introduce palabras que permiten afirmar que conoce la existencia de un cáncer, pero no podemos afirmar que conoce la situación de enfermedad avanzada o el grado de afectación de este ni el pronóstico.

Categoría D: El paciente conoce diagnóstico de enfermedad tumoral maligna avanzada. Las respuestas obtenidas permiten afirmar que conoce la existencia de un cáncer en situación avanzada pero no permiten afirmar que conozca o sospeche la proximidad de la muerte cuando este fuera el caso (es decir, si realmente está en fase terminal)

Categoría E: EL paciente conoce diagnóstico de enfermedad tumoral en situación terminal. El discurso del paciente orienta al pleno conocimiento de su situación real.

\section{Ejemplos para la valoración de la entrevista}

\begin{tabular}{|l|l|}
\hline $\begin{array}{l}\text { no sé } \\
\text { pregunte a mi familia } \\
\text { tuve un tumor, pero ya estoy curado, ya no tengo enfermedad } \\
\text { el paciente no aborda el tema durante la entrevista. }\end{array}$ & Categoría A \\
\hline $\begin{array}{l}\text { un tumor, un bulto, una masa } \\
\text { una manchita } \\
\text { una inflamación, una infección } \\
\text { estoy enfermo de... "cita el órgano afectado" }\end{array}$ & Categoría B \\
\hline
\end{tabular}




\begin{tabular}{|c|c|}
\hline $\begin{array}{l}\text { cáncer, neoplasia, enfermedad maligna, tumor maligno x } \\
\text { el bicho dentro x } \\
\text { una enfermedad incurable } \\
\text { algo malo en.... "cita el órgano afectado" } \\
\text { un tumor que está operado y controlado } \\
\text { un tumor que está respondiendo o mejorando con los tratamientos } \\
\text { médicos } \\
\text { un tumor, pero tengo esperanzas de curar o volver a operar } \\
\text { estoy a tratamiento de quimioterapia, } \\
\text { recibo o he recibido radioterapia } \\
\text { Necesito tratamiento de hormonas }\end{array}$ & Categoría C \\
\hline $\begin{array}{l}\text { tengo metástasis } \\
\text { un tumor en estadio IV o situación avanzada x } \\
\text { el tumor sigue progresando, avanzando, se extendió } \\
\text { los tratamientos médicos no han dado resultado } \\
\text { el tumor volvió a aparecer tras la cirugía } \\
\text { el tumor permanece, pero está dormido, controlado **** } \\
\text { la evolución ha sido mala } \\
\text { necesito tratamiento para que la enfermedad se haga crónica } \\
\text { Tengo un tratamiento paliativo, }\end{array}$ & Categoría D \\
\hline $\begin{array}{l}\text { todo esto no valió para nada } \\
\text { esto es el final } \\
\text { voy a morir } \\
\text { no me queda mucho } \\
\text { hay que despedirse } \\
\text { ya no me ponen ningún tipo de tratamiento } \\
\text { tengo un tratamiento para retrasar lo inevitable, retrasar la muerte } \\
\text { tengo un tratamiento para vivir más, vivir mejor, lo que se pueda, }\end{array}$ & Categoría E \\
\hline
\end{tabular}


